\title{
EDITORIAL
}

\section{Introduction to 'All roads take to the brain: neural control of energy homeostasis in health and disease'}

\author{
International Journal of Obesity (2016) 40, 191-192; doi:10.1038/ \\ ijo.2015.245
}

The rapidly increasing incidence of obesity-related health problems in ever-younger patients in industrialized countries, the consequent adverse impact on the quality of life and the attendant dramatic rise in health-care costs have focused attention on our poor understanding and inability to deal effectively with disordered eating and body weight regulation. This is also reflected by an increase in scientific efforts to unravel the mechanisms that regulate energy balance and to identify potential targets for a pharmacotherapy of obesity. On the other side of the energy balance equation are the anorexia and cachexia that accompany chronic diseases. Although clinically distinct, in recent years it has become increasingly clear that the underlying disturbances in the regulation of energy homeostasis, despite their differences, share certain key features. Thus, it is now widely acknowledged that metabolic perturbations are key pathogenic mechanisms involved in obesity and responsible for the phenotypic expression of different acute and chronic diseases. The complex controls of energy intake and expenditure (metabolism) have been extensively investigated, but a comprehensive view of the sequential molecular and metabolic changes that lead to obesity or the clinical metabolic features in human disease is still lacking. Of specific scientific and clinical interest is therefore research into a better understanding of the mechanisms linking and coordinating the complex responses occurring at different levels (that is, molecular, tissue and organs). The nervous system coordinates the metabolic and clinical responses to internal and external insults with the aim of maintaining energy homeostasis, which is essential for metabolic health and survival. This is achieved through neuronal circuits that control energy intake and expenditure as well as physiological processes affecting metabolism. Neurons throughout the CNS, but highly concentrated in some specific brain areas, sense humoral and neural signals of systemic energy status and relay this information to secondary neurons that regulate physiological processes so as to maintain energy homeostasis. The pertinent neuronal circuits must integrate information about short-term changes in nutrient availability in the context of long-term energy status. For example, the same signal presumably triggers different responses in the fasted versus fed condition. This requires mechanisms that rapidly and reversibly adjust the responsiveness of the involved neuronal circuits to changes in nutrient availability.

The workshop titled 'All roads take to the brain-neural control of energy homeostasis in health and disease,' which was held during 21-26 September 2014, in Ascona, Switzerland, at the Centro Stefano Franscini of the Swiss Federal Institute of Technology (ETH) addressed these issues. Throughout an exciting week of seminars and discussions, the workshop brought together around 70 researchers and clinicians from around the world who attempted to evaluate and integrate many aspects that are important for understanding the bidirectional interactions between the periphery and the brain in the control of eating and energy balance, their physiological and pathological implications and how they are best studied in animal models as well as in humans. Also, the workshop clearly succeeded in treating the topic comprehensively, from molecular biology to the clinic. The reviews collected in this special section of the International Journal of Obesity reflect the workshop's synthetic and translational spirit and provide a representative overview of those presentations and discussions that were focused on human and more clinically oriented studies, whereas the exclusively basic science-oriented presentations will be published as a series of invited reviews elsewhere. While many of the authors illuminate various and sometimes overlapping aspects of the unifying theme of the workshop through the prisms of their own special expertise, they nonetheless also provide excellent overviews of this important emerging field.

Morselli et al. ${ }^{1}$ highlight sexual dimorphismic response to chronic high-fat diets. They emphasize that, although obesity affects both males and females, there is a sexual dimorphism in the development of metabolic complications. Premenopausal women have less metabolic co-morbidities associated with obesity than men; however, the prevalence of obesity and diabetes increases dramatically in postmenopausal women. They review studies that have confirmed the importance of the estrogen receptor- $a$ (ER- $a$ ) in the central nervous system (CNS); ER-a functions in the hypothalamus to regulate food intake, glucose homeostasis and energy expenditure. Furthermore, they show epidemiological evidence demonstrating that premenopausal females are protected from the adverse effects of obesity. The findings that are actually present suggest that estradiol/ER-a has a role in the control of obesity-associated metabolic complications.

Steinert et al. ${ }^{2}$ deal with intestinal glucagon-like peptide-1 (GLP-1) and satiation, from man to rodents and back. In response to luminal food stimuli during meals, enteroendocrine cells release gastrointestinal $(\mathrm{Gl})$ peptides that have long been known to control secretory and motor functions of the gut, pancreas and liver. GLP-1 has emerged as one of the most important GI peptides because of a combination of functions not previously ascribed to any other molecule. GLP-1 potentiates glucose-induced insulin secretion, suppresses glucagon release, slows gastric emptying and may serve as a satiation signal, although the physiological status of the latter function has not been fully established yet. The available evidence for intestinal GLP-1 to fulfill a number of established empirical criteria for assessing whether a hormone inhibits eating by eliciting physiological satiation in man and rodents is reviewed.

Gatta-Cherifi and $\operatorname{Cota}^{3}$ give an overview of new insights into the role of the endocannabinoid system (ECS) in the regulation of energy balance. They discuss recent advances and novel therapeutic options targeting the ECS that might prove to be successful for the treatment of obesity and associated metabolic disorders. The ECS is an important lipid signaling system critically involved in the regulation of energy balance, since it exerts a regulatory control on essentially every aspect related to the search, the intake, the metabolism and the storage of calories. An overactive endocannabinoidcannabinoid type 1 (CB1) receptor signaling promotes the development of obesity, insulin resistance and dyslipidemia, representing a valuable pharmacotherapeutic target for obesity and metabolic disorders. However, due to psychiatric side effects, the first generation of brain-penetrant CB1 receptor blockers developed as anti-obesity treatment were removed from the European market in 2009. Since then the 
mechanisms of action of the ECS in energy balance and metabolism have been investigated with the aim of generating new knowledge that would allow targeting this system in a more specific and selective manner.

Finally, Tsai et al. ${ }^{4}$ discuss anorexia/cachexia and obesity as two sides of the same coin. They highlight the role of the transforming growth factor- $\beta$ superfamily cytokine MIC-1/GDF15. Anorexia/cachexia usually occurs as a complication of disease processes and, by definition, its major characteristic is loss of muscle mass, but also loss of adipose tissue. Loss of muscle also occurs as part of normal aging, and in some individuals this can be severe enough to lead to cachexia. Anorexia/cachexia occurs in a number of disease processes such as chronic renal, liver and cardiac failure, and in fact most commonly in advanced cancer. They discuss the available data linking anorexia/cachexia syndromes to their mechanisms of central appetite regulation, and suggest a potential target for therapy of cancer.

In sum, the papers in this special issue of the International Journal of Obesity present a multifaceted approach to a fascinating, rapidly developing and important area of research. We hope the readers will find this an interesting, timely and useful contribution to the ongoing discussion of obesity and anorexia/ cachexia as a scientific challenge as well as a social and clinical problem.

\section{ACKNOWLEDGEMENTS}

We gratefully acknowledge the Congressi Stefano Franscini (ETH Zurich), the Swiss Academy of Sciences and the Swiss National Science Foundation, as well as TSE, Fresenius Kabi, Research Diets, PepsiCo, Boehringer Ingelheim and Bucher Biotec who provided financial and material support for the workshop. We thank all contributing authors for their efforts. Last but not least, we thank Dr N Dhurandhar and Dr R Atkinson, as the responsible editors of the International Journal of Obesity, for giving us the opportunity to publish the papers from this workshop in this special issue.

W Langhans ${ }^{1}, \mathrm{H}-\mathrm{R}$ Berthoud ${ }^{2}$ and $\mathrm{M}$ Westerterp-Plantenga ${ }^{3}$ ${ }^{1}$ Institute of Food, Nutrition and Health, ETH Zurich, Schwerzenbach, Switzerland;

${ }^{2}$ Pennington Biomedical Research Center, Baton Rouge, LA, USA and ${ }^{3}$ Maastricht University, Maastricht, The Netherlands E-mail: Wolfgang-langhans@ethz.ch

\section{REFERENCES}

1 Morselli E, Frank AP, Rodriguez-Navas C, Criollo A, Clegg DJ. Sexual dimorphismic response to chronic high fat diet. Int J Obes 2015.

2 Steinert RE, Beglinger C, Langhans W. Intestinal GLP-1 and satiation-from man to rodents and back. Int J Obes 2015.

3 Gatta-Cherifi B, Cota D. New insights on the role of the endocannabinoid system in the regulation of energy balance. Int J Obes 2015.

4 Tsai Vicky WW, Lin Shu, Brown David A, Salis Amanda, Breit Samuel N. Anorexia/cachexia and obesity are two sides of the same coin. Role of the TGF-b superfamily cytokine MIC-1/GDF15. Int J Obes 2015. 